\title{
Rotation Effect of In-plane FM layer on IrMn Based GMR-SV Film
}

\author{
Purevdorj Khajidmaa ${ }^{1}$, Jong-Gu Choi ${ }^{2}$, and Sang-Suk Lee ${ }^{1,2 *}$ \\ ${ }^{1}$ Department of Oriental-Western Biomedical Engineering, General Graduate School, Sangji University, Wonju 26339, Korea \\ ${ }^{2}$ Department of Oriental Biomedical Engineering, Sangji University, Wonju 26339, Korea
}

(Received 19 December 2016, Received in final form 2 January 2017, Accepted 3 January 2017)

\begin{abstract}
The magnetoresistance (MR) properties of antiferromagnetic (AFM) IrMn based giant magnetoresistance-spin valve (GMR-SV) was investigated in view point of the artificial rotation effect of ferromagnetic (FM) layer in the plane induced by an applied field during the post annealing temperature. The MR curves measured with an azimuthal angle region of $\phi=0^{\circ}-360^{\circ}$ are depended on the annealing temperature and the magnetization easy axis of two free NiFe layers and two pinned NiFe layers in dual-type GMR-SV film. Especially, the annealing temperature and sample rotation angle $(\theta)$ maintained to the magnetic sensitivity (MS) of $1.4 \% / O e$ with an isotropic region angle of $110^{\circ}$ are $100^{\circ} \mathrm{C}$ and $90^{\circ}$, respectively.
\end{abstract}

Keywords : dual-type giant magnetoresistance-spin valve (GMR-SV) film, annealing temperature, rotation angle, coercivity $\left(\mathrm{H}_{\mathrm{c}}\right)$, magnetic sensitivity (MS)

\section{Introduction}

A giant magnetoresistance-spin valve (GMR-SV) sensor composed of antiferromagnetic (AFM)/ferromagnetic (FM)/ nonmagnetic/FM multilayer has been used as a signal recycling head of the high density hard disk drive. The application development of one highly sensitive nano magnetic thin film style can be applied to medical sensor chip using magnetic particles, bio-magnetic measurement diagnostic sensor, and ultra-fine magnetic field measurement sensor [1-3]. Also, a highly sensitive magnetic field sensor was utilized widely in linear or rotary encryption, proximity detector, velocity sensor, position sensor, and geomagnetic field magnetometer. A GMR-SV sensor has developed to be enabling to utilize into bio-fusion technology division along with the study about much enhanced the magnetoresistance (MR) ratio and the magnetic sensitivity (MS) $[4,5]$.

Meanwhile, the convergence study of nano-technology and bio-technology has developed the usage of GMR-SV element. It has been available as biosensor and is causing great concern among one of the various applications as medical sensor which is inspecting bio-magnetic field [6]. While selecting specific species with the size of mole-

(C)The Korean Magnetics Society. All rights reserved.

*Corresponding author: Tel: +82-33-730-0415

Fax: +82-33-738-7610, e-mail: sslee@sangji.ac.kr cular level, bio-sensor using GMR-SV would be available to observe biological cells using magnetic signal or the movement of chemical molecular. The investigation in detail responses and activities of bio-particles with super paramagnetic bead attached to molecular cell would be very essential to life and medicine [7]. Therefore, it would be needed to have optimal production conditions of multilayer thin film which has a high MS to develop GMR-SV element applying the optical lithography process technology [8-10].

As using optical lithography process to consider dependence of width, length and thickness about a magnetic sensitivity of GMR-SV element, the pinned layer and the free layer should be set a width direction as easy axis and a length direction as hard axis, respectively. It would observed as decrease of MR ratio. The shape magnetic anisotropy means that the length of element gets 10 times bigger than the width to be formed to easy axis in length direction [11]. As micron-sized magnetic bead has limited isotropy, the condition as GMR-SV bio-element detecting super paramagnetic characteristics should have a high MS with the wide rotational domain.

Generally, to utilize as biosensor based on the AFM IrMn or FeMn layers/FM multilayer structure, it will fabricate a highly sensitive GMR-SV films making NiFe or $\mathrm{CoFe}$ ferromagnetic layer into the pinned and free layer. In order to enhance the MS considering the properties of soft magnetic thin film, the GMR-SV multilayer should 
be optimized the thickness of $\mathrm{Cu}$ spacer $[12,13]$.

This study refers the methods to enhance isotropic MR ratio of dual-type GMR-SV film element placing IrMn of AFM substance in the middle, adjacent ferromagnetic layer into lower pinned layer and upper pinned layer. In order to be crossed pinned layer on the surface and free layer's easy axis direction, the post annealing treatment performed in vacuum [12]. The control of magnetization direction according to annealing temperature having the spin status of magnetic domains moved to the direction of applied magnetic field. When all magnetic layers make thin film for dual type GMR-SV, the pinned layer and the free layer induced to uniaxial anisotropy without a length or a width direction. The isotropic MR properties analyzed by the measurement of MR curve of GMR-SV film applied with an optimum post annealing treatment.

\section{Experimental Method}

The dual-type GMR-SV film of Corning glass (7059)/ $\mathrm{Ta} / \mathrm{NiFe} / \mathrm{Cu} / \mathrm{NiFe} / \mathrm{IrMn} / \mathrm{NiFe} / \mathrm{Cu} / \mathrm{NiFe} / \mathrm{Ta}$ multilayer was fabricated using the dc magnetron sputtering deposition system. We used membrane made of stainless panel to prevent confusion between mutual interference of plasma or target elements while depositing independently $\mathrm{Ta}$, $\mathrm{NiFe}, \mathrm{Cu}$, IrMn of round shaped 3 inch 4 targets as dc magnetron sputtering system [11-13]. All of GMR-SV multilayers were consisted of the antiferromagnetic IrMn layer and the ferromagnetic $\mathrm{NiFe}$ layer as the role of pinned layer and free layer, the spacer $\mathrm{Cu}$ layer, and the bottom and top Ta layers. The deposition rates of Ta, $\mathrm{NiFe}, \mathrm{Cu}, \mathrm{IrMn}$ thin films were $0.1 \mathrm{~nm} / \mathrm{s}, 0.15 \mathrm{~nm} / \mathrm{s}, 0.12$ $\mathrm{nm} / \mathrm{s}$, and $0.1 \mathrm{~nm} / \mathrm{s}$, respectively. For GMR-SV multilayer structure, the optimized thicknesses for Ta layer, $\mathrm{NiFe}$ layer, $\mathrm{Cu}$ layer, $\mathrm{NiFe}$ layer, and $\mathrm{IrMn}$ layer are $5 \mathrm{~nm}, 8$ $\mathrm{nm}, 2.3 \mathrm{~nm}, 4 \mathrm{~nm}$, and $5 \mathrm{~nm}$, respectively.

We installed $0.3 \mathrm{~mm}$ thickness of shadow metal mask between permanent magnet of both sides on glass substrate during the deposition of multilayer thin film, as shown in Fig. 1(a). A magnitude of center magnetic field was 350 Oe. The feature and structure of dual-type GMRSV film sample is patterned with $0.6 \mathrm{~mm}$ of width and 15 $\mathrm{mm}$ length after depositing over one substrate, as shown in Fig. 1(a).

The constant current flowing at both side of electrodes to read output voltage which is occurred from 2 electrodes with constant frequency to substitute to Ohm's law to obtain the value of MR put in the electromagnet. The magnetic field to be placed to vertical direction with electric current applied to sample and the value of $\mathrm{dc}$ electric current permitted to pattern was $1 \mathrm{~mA}$. To obtain major MR curve during the measuring major MR loop, as shown in Fig. 1(b), the value of magnetic field marked to Gauss Meter was from +1200 Oe to -1200 Oe. The frequency of function generator which can determine the number of measurement data and resolution was 1.7 $\mathrm{mHz}$. To obtain one of the measuring minor MR loop from -100 Oe to +100 Oe, measurement frequency was $0.5 \mathrm{mHz}$.

Figure 1(b) shows the major MR curve for a dual-type IrMn based GMR-SV film with MR ratio $=6.5 \%, \mathrm{MS}=$ $1.3 \% / \mathrm{Oe}$, and coercivity $\left(\mathrm{H}_{\mathrm{c}}\right)=3.0$ Oe which are measured at minor MR curve. The circle numbers (1), (2), (3), (4), (5) marked to major MR curve of Fig. 1(b) are represented by the different five configuration array steps of FM and AFM layers according to the applied measuring magnetic field from +1200 Oe to -1200 Oe. Configuration array for the magnetic spins of FM and AFM layers detailed explained in Fig. 3 [14, 15].

As core technology of this study, in order to be crossed
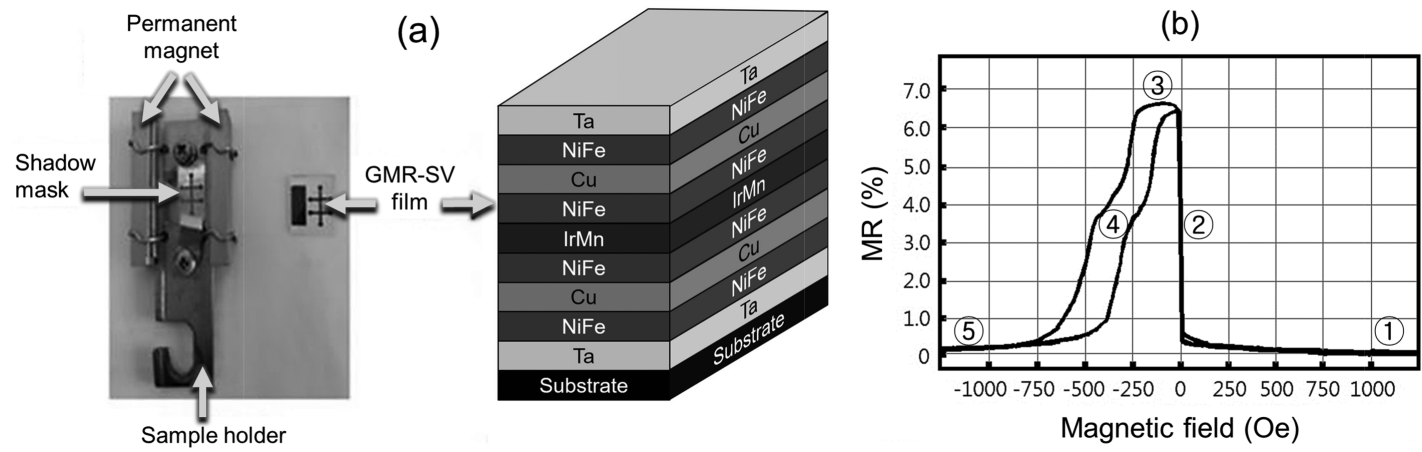

Fig. 1. (a) The shadow metal mask, the permanent magnet, and the patterning dual-type GMR-SV multilayer sample having a width of $0.6 \mathrm{~mm}$ and a length of $15 \mathrm{~mm}$. Schematic of the sample structure for the dual-type GMR-SV multilayer. (b) Major MR curve for a dual-type IrMn based GMR-SV film with MR ratio $=6.5 \%, \mathrm{MS}=1.3 \% / \mathrm{Oe}$, and $\mathrm{H}_{\mathrm{c}}=3.0$ Oe which are measured at minor MR curve. Here, the circle numbers (1), (2), (3), (4), (5)) are represented by the different five configuration array steps of FM and AFM layers according to the applied measuring magnetic field from +1200 Oe to -1200 Oe. 


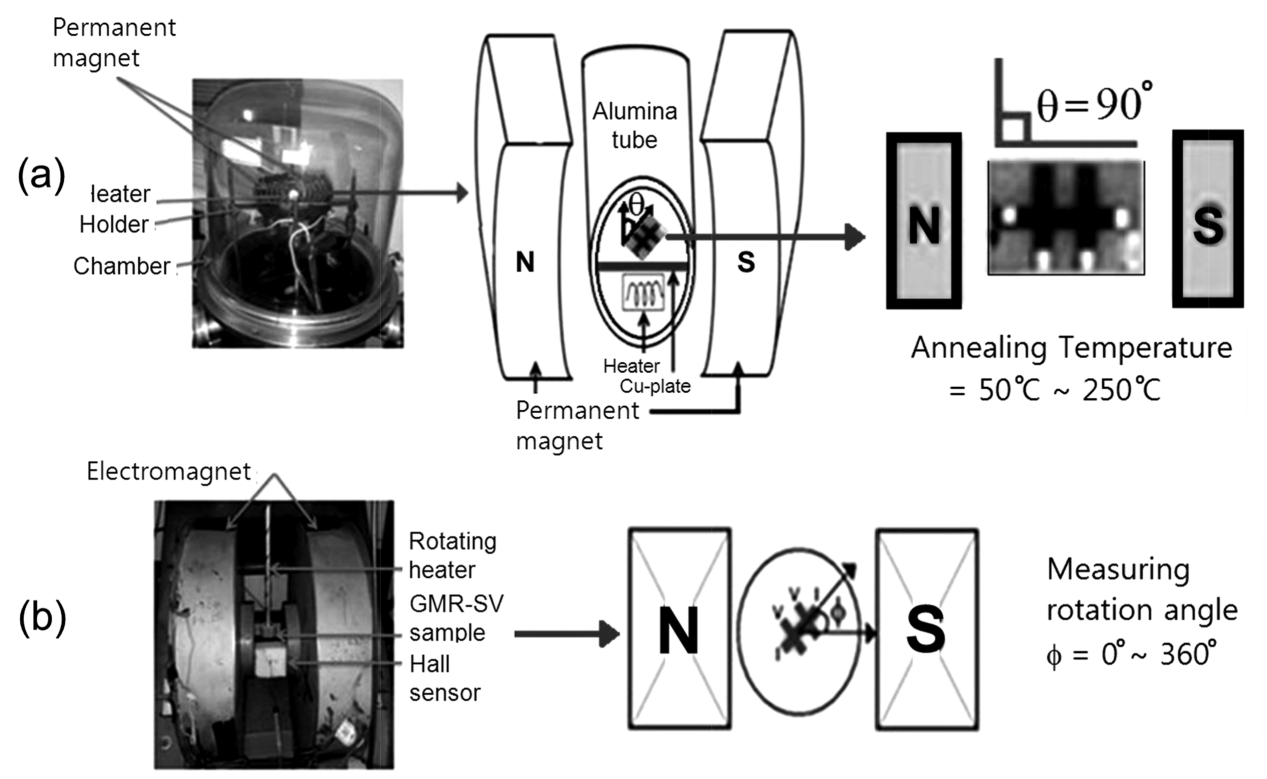

Fig. 2. (a) Electromagnet and heater inside of the vacuum chamber for the annealing treatment. The detailed form of annealing heating system composed of chamber, lamp, heater, and permanent magnet. Here $\theta$ is a rotation angle of sample between the applied perpendicular direction during the post annealing and the hard axis performed the deposition of the dual-type GMR-SV. (b) The electromagnet, sample holder, and Hall sensor for measuring the curve of MR versus magnetic field. The measurement of MR curve depending on an azimuthal angle $\phi$ between measuring magnetic field axis and uniaxial easy axis.

free layer and pinned layer we fixed a sample by applying magnetic field of permanent magnet during the vacuum post annealing treatment in plan of GMR-SV multilayer, as shown in Fig. 2(a). This method can be rotated a thin film sample into random angle when we proceed annealing treatment in vacuum chamber. We kept $5 \times 10^{-6}$ Torr of chamber vacuum while heat treatment. A magnetic field that can induce uniaxial anisotropy while vacuum annealing treatment was 350 Oe like condition for one of GMR-SV deposition. We installed the halogen lamp wrapped with a cylindrical alumina in the center of several round ferrite permanent magnets symmetrically, as shown in Fig. 2(a).

We input thin film sample in the alumina ceramic cylinder wrapped with flat $\mathrm{Cu}$ panel which can keep consistent temperature because they can receive direct radiant heat of operating lamp. The $\theta$ noticed in Fig. 2(a) is the rotation angle of sample between the applied perpendicular direction of magnetic field during the post annealing and the hard axis performed the deposition of the dual-type GMR-SV [9]. We implemented annealing treatment of thin film sample on the flat panel of $\mathrm{Cu}$. Increasing to annealing temperature at $5{ }^{\circ} \mathrm{C} / \mathrm{m}$ speed, the sample stayed for $1 \mathrm{hr}$ at goal temperature. After it reached to target temperature at consistent rising speed and decreased to room temperature at $2{ }^{\circ} \mathrm{C} / \mathrm{m}$ speed.

Figure 2(b) shows a real 4-probe MR system and a schematic diagram which can be measured MR curve about a dual-type GMR-SV induced to uniaxial anisotropy magnetic field after annealing treatment. The sample rotated according to the azimuthal angle $\phi=0^{\circ}-360^{\circ}$ of sample maintained to $0^{\circ}$ angle of the applied magnetic field direction. In order to observe magnetic resistance properties of thin film sample, we determined coercivity $\mathrm{H}_{\mathrm{c}}(\mathrm{Oe})$, exchange coupling field $\mathrm{H}_{\mathrm{ex}}(\mathrm{Oe})$, MR ratio (\%) from MR curve which was measured from 4 electrodes magnetic resistance measurement system at room temperature individually. For round panel which is used as holder while measuring, we set the azimuthal angle $\phi$ between the length direction of thin film of sample surface and the applied magnetic field direction to be adjustable from $0^{\circ}$ to $360^{\circ}$.

\section{Results and Discussion}

Figure 3(a) shows configuration array of typical magnetic spins for each layer of the dual-type GMR-SV film having $\mathrm{FM} / \mathrm{Cu} / \mathrm{FM} / \mathrm{AFM} / \mathrm{FM} / \mathrm{Cu} / \mathrm{FM}$ system, as mentioned in Fig. 1(b). Figure 3(b) show the schematic of spin structure at five different points marked to the major MR curve in Fig. 1(b).

Five different configuration array steps of FM and AFM layers are explained as following contents [14, 15]. (1) : parallel to the right direction for four spins of FM 
(a)

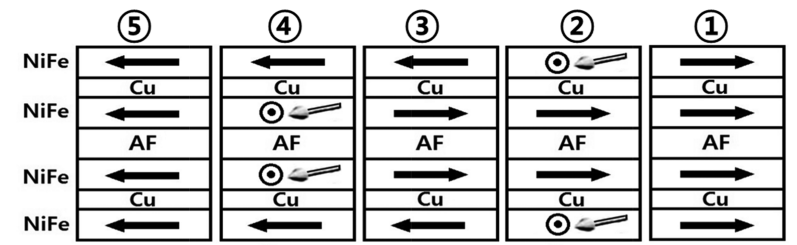

(b)

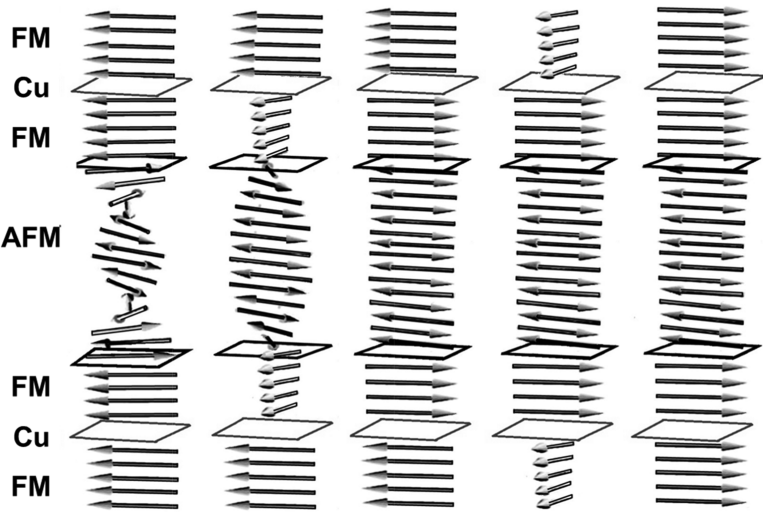

Fig. 3. (a) The configuration array of typical magnetic domain (b) the schematic of spin structure at five different points marked to the major MR curve in Fig. 1(b). The creation of a partial AFM domain of IrMn layer wall and the spins close to the FM/AFM/FM interface can see in the center of a dual-type GMR-SV multilayer.

layers, (2) : the $90^{\circ}$ direction for two spins of free FM layers and parallel to the right direction for two spins of pinned FM layers, (3) : antiparallel to the right direction for two spins of free FM layers and parallel to the right direction for two spins of pinned FM layers, (4) : antiparallel to the right direction for two spins of free FM layers and antiparallel to the $90^{\circ}$ direction for two spins of pinned FM layers, and (5) : antiparallel to the right direction for four spins of FM layers.

Figure 4(a) refers to the major MR curves that measured with the same length direction after vacuum annealing treatment at six different temperatures from as grown to $150{ }^{\circ} \mathrm{C}$ during a staying time of $1 \mathrm{hr}$. All samples did annealed at a rotation angle of $\theta=90^{\circ}$ between an uniaxial direction and an applied field under the annealing treatment, as shown in Fig. 2(a). The minor MR curves of a dual-type IrMn based GMR-SV films measured after six different post annealing temperatures, as shown in Fig. 4(b). The measurement angles of MR curves are $\phi=$ $0^{\circ}$, as shown in Fig. 2(b). Each MR ratio dual-type GMRSV film sample obtained from minor MR curve for the easy axis induced to uniaxial anisotropy.

We had $5{ }^{\circ} \mathrm{C} / \mathrm{m}$ of temperature increasing rate in order to be reached to the goal temperature. A, B, C, D, E, and $\mathrm{F}$ as six annealing temperatures marked in Fig. 4(a) and Fig. 4 (b) are coincided to as grown, $50^{\circ} \mathrm{C}, 75^{\circ} \mathrm{C}, 100^{\circ} \mathrm{C}$,

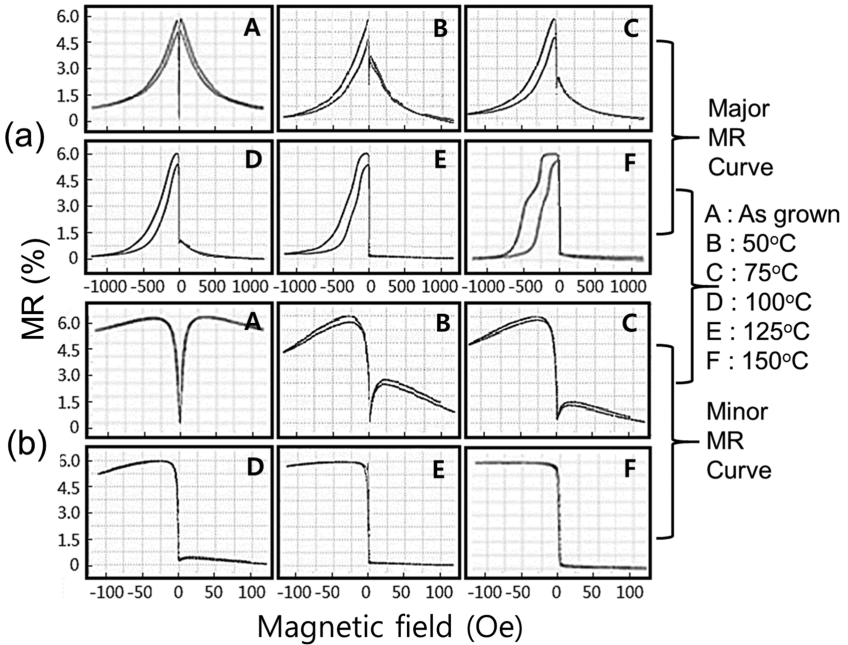

Fig. 4. (a) Major MR curves and (b) minor MR curves of a dual-type IrMn based GMR-SV films measured after six post annealing temperatures from as grown to $150{ }^{\circ} \mathrm{C}$ during a staying time of $1 \mathrm{hr}$ and a rotation angle of $\theta=90^{\circ}$ between an uniaxial direction and an applied field under the annealing treatment. The measurement angles of MR curves are $\phi=0^{\circ}$. Here, $\mathrm{A}, \mathrm{B}, \mathrm{C}, \mathrm{D}, \mathrm{E}$, and $\mathrm{F}$ are coincided to as grown, $50^{\circ} \mathrm{C}$, $75^{\circ} \mathrm{C}, 100{ }^{\circ} \mathrm{C}, 125^{\circ} \mathrm{C}$, and $150^{\circ} \mathrm{C}$, respectively.

$125^{\circ} \mathrm{C}$, and $150{ }^{\circ} \mathrm{C}$, respectively. After being stayed for $1 \mathrm{hr}$ in annealing temperature, we lowered temperature descending rate with $5{ }^{\circ} \mathrm{C} / \mathrm{m}$ of speed which is same as increasing rate to the room temperature. As compactness between interfaces due to vacuum annealing treatment effect, the value of surface resistance measured by 4-probe get shrunk to roughly $0.5 \Omega-0.7 \Omega$. Because it would go through annealing treatment process under flat outer magnetic field, it had $\mathrm{H}_{\mathrm{c}}$ and $\mathrm{H}_{\mathrm{ex}}$ accompanied with a good thermal stability of magnetic multilayer. We can predict that MR property would drop steadily if the post annealing treatment temperature is more than $150{ }^{\circ} \mathrm{C}$. On the other hand, the major and minor loops of Fig. 4 are showing a typical MR property of dual-type GMR-SV.

For multilayer structure, it is considered to show a phenomenon that MR ratio and magnetic properties are interfered due to mutual exchange coupling of pinned layer and free layer with a space $\mathrm{Cu}$ layer and a roughness of interface. From the major and minor MR curves of Fig. 4, we can know that the different MR curves of major loop measured from around 0 Oe to \pm 1200 Oe have anisotropic magnetoresistance (AMR) effect. The values of coercivity of 0 Oe at hard axis AMR curve (A) differently increased to 3.2 Oe at easy axis MR curve (A). As the annealing temperature (AT) increased A to F, the hard axis MR curve of A changed to the easy axis of MR curve of $F$ and MR ratio was almost maintained to the 
similar value having a about $6.0 \%$. The values of MS and $\mathrm{H}_{\mathrm{c}}$ of minor MR curve $\left(\mathrm{D}\right.$; $\left.\mathrm{AT}=100{ }^{\circ} \mathrm{C}\right)$ are $1.4 \% / \mathrm{Oe}$ and 1.0 Oe. In case of minor MR curve $\left(\mathrm{F} ; \mathrm{AT}=150^{\circ} \mathrm{C}\right)$, those are $1.3 \% / \mathrm{Oe}$ and $3.2 \mathrm{Oe}$. From these results, an optimized annealing temperature when easy axis of magnetic layer rotates on floor plan for the dual-type GMRSV having $\mathrm{Ta}(5 \mathrm{~nm}) / \mathrm{NiFe}(8 \mathrm{~nm}) / \mathrm{Cu}(2.3 \mathrm{~nm}) / \mathrm{NiFe}(4 \mathrm{~nm}) /$ $\operatorname{IrMn}(5 \mathrm{~nm}) / \mathrm{NiFe}(4 \mathrm{~nm}) / \mathrm{Cu}(2.3 \mathrm{~nm}) / \mathrm{NiFe}(8 \mathrm{~nm}) / \mathrm{Ta}(5$ $\mathrm{nm})$ induced by uniaxial anisotropy magnetic field was $100{ }^{\circ} \mathrm{C}$.

Especially, during the vacuum annealing treatment, to rotate dual GMR-SV film which has easy axis as width direction at $90^{\circ}$ angle to place hard axis to the direction of easy axis, we done post annealing treatment process under $350 \mathrm{Oe}$ of flat magnetic field during $1 \mathrm{hr}$. The major and minor MR curves for $\mathrm{A}$ in Fig. 3 refers to those which were measured after annealing treatment at temperature $100^{\circ} \mathrm{C}$ with rotating $45^{\circ}$ section from $0^{\circ}$ to $+90^{\circ}$ for the applied magnetic field direction.

For easy axis of dual GMR-SV film sample which uniaxial anisotropy is induced, each MR ratios of minor loops for one cycle measurement which was measured with interval angle of $22^{\circ}-23^{\circ}$ keep the value of around $6.0 \%-6.5 \%$, as shown in Fig. 5. Especially, MR curve measured at $\phi=45^{\circ}$ angle, the thickness of dual-type structure mainly with IrMn layer have same symmetric value between GMR-SV film of lower layer and GMRSV film of upper layer. The $\mathrm{H}_{\mathrm{c}}$ and $\mathrm{H}_{\mathrm{ex}}$ of upper and lower layer get decreased 0.5 times than that of lower layer. From MR curve of minor loop that outer magnetic field measured from 0 Oe to around $\pm 100 \mathrm{Oe}$, we could find out that the value of $\mathrm{H}_{\mathrm{c}}$ get maintained to $1.0 \mathrm{Oe}$ at $\phi$ $=0^{\circ}$ compared to annealing treatment at $150{ }^{\circ} \mathrm{C}$.

It refers to major and minor MR curves that were measured to induce uniaxial anisotropy as length direction with a changed $45^{\circ}$ angle differently on the thin film plan by a vacuum annealing treatment. Therefore, Fig. 5 shows that the similar two minor MR curves at $\phi=45^{\circ}$ and $\phi=$ $225^{\circ}$ refers to the status with a changed $45^{\circ}$ angle in-plane FM layer of the dual-type IrMn based GMR-SV films.

From MR properties of a dual-type GMR-SV film sample done with annealing treatment at $100{ }^{\circ} \mathrm{C}$ of temperature, we can predict that IrMn layer of antiferromagnetic substance and $\mathrm{NiFe}$ pinned layer move slowly to the exchange combined spin direction and to spin direction of free layer NiFe. Figure 5 synthetically refers that dual GMR-SV film sample with isotropy MR properties has MS relied on one annealing treatment temperature of 100 ${ }^{\circ} \mathrm{C}$. Figure 5 show that two regions of azimuthal angles of isotropic MS for the samples of annealing temperature of $100^{\circ} \mathrm{C}$ are $\phi=-10^{\circ}-100^{\circ}$ and $\phi=170^{\circ}-280^{\circ}$.

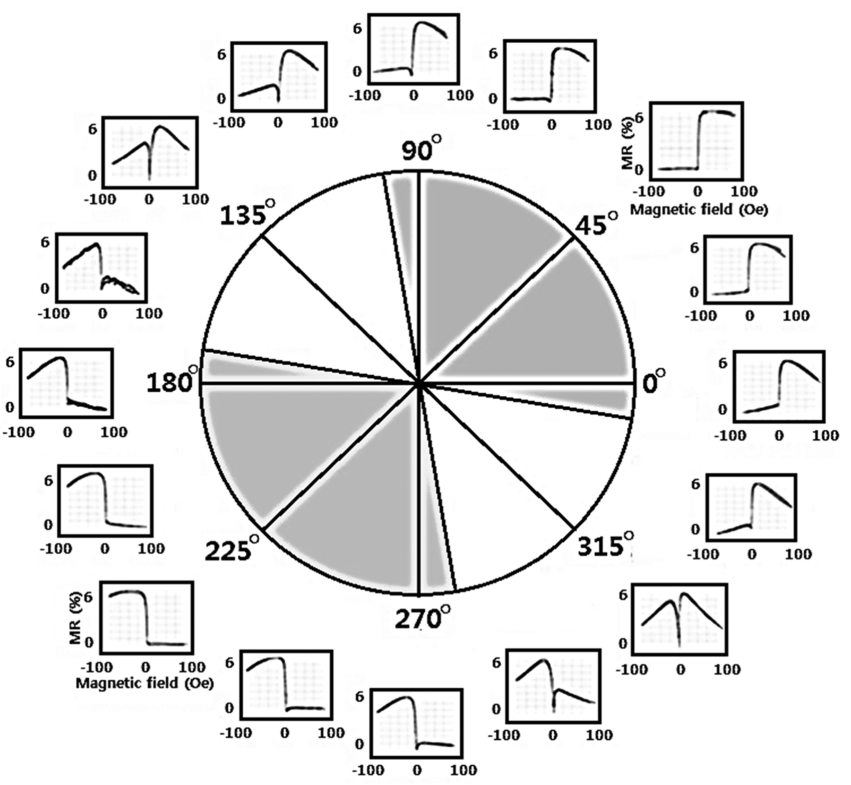

Fig. 5. Minor curves of $M S, H_{c}$, and MR ratio versus the magnetic field $( \pm 100 \mathrm{Oe})$ after a post annealing temperatures of $100{ }^{\circ} \mathrm{C}$ and a rotation angle of $\theta=90^{\circ}$. The similar two minor MR curves at $\phi=45^{\circ}$ and $\phi=225^{\circ}$ refers to the status with a changed $45^{\circ}$ angle in-plane FM layer of the dual-type IrMn based GMR-SV films. The region of isotropic distribution of MS depending on the measurement azimuthal angles is $\phi=$ $-10^{\circ}-100^{\circ}$ and $\phi=170^{\circ}-280^{\circ}$.

When we compared with minor loops at $\phi=0^{\circ}$, as shown in Fig. 5, we can find out that the saturation value of MR ratio is not consistent to be decreased as magnetic field is over \pm 5 Oe. Around 0 Oe it shows a consistent MS obtained from linear MR curve with a few hysteresis property. For the magnetic field from $0^{\circ}$ to $90^{\circ}$ of rotating angle of thin film sample after the annealing temperature of above $150{ }^{\circ} \mathrm{C}$, a measuring MR is same with rotating angle of thin film to be completed to minor loop, which has the same isotropy MR curve individually.

As shown in Fig. 6, we compared and analyzed three minor MR curves that were measured after an annealing treatment temperature of as grown, $100{ }^{\circ} \mathrm{C}$, and $150{ }^{\circ} \mathrm{C}$

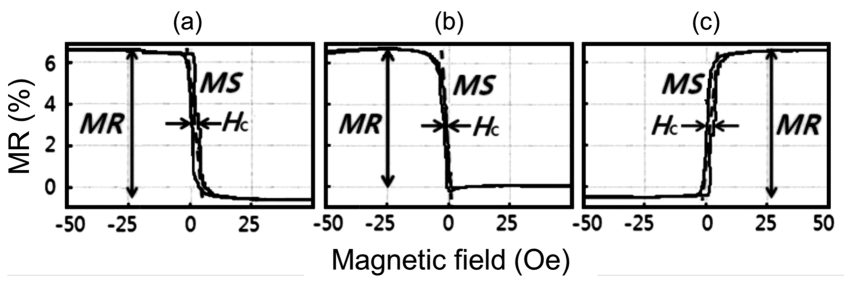

Fig. 6. Minor MR curves of $M S, \mathrm{H}_{c}$, and $M R$ ratio versus the magnetic field $( \pm 50 \mathrm{Oe}$ ) for the dual-type IrMn based GMRSV films after a post annealing temperatures of (a) as grown, (b) $100{ }^{\circ} \mathrm{C}$, and (c) $150{ }^{\circ} \mathrm{C}$ at the rotation angle of $\theta=90^{\circ}$. 
with a rotation angle of $\theta=95^{\circ}$. For as grown and $150^{\circ} \mathrm{C}$ of annealing treatment temperatures, MS shows $1.3 \% / \mathrm{Oe}$ of value as shown in Fig. 1(b), Fig. 6(a), and Fig. 6(c). The direction placing thin film sample with annealing treatment in Fig. 2(a) in length direction is corresponding to the applied magnetic field to rotate $90^{\circ}$.

The MR ratio, $\mathrm{H}_{c}$, and MS which were calculated from MR curve measuring $360^{\circ}$ with whole rotating interval were shown to circular diagram as Fig. 5. Placing thin film sample along with width direction corresponding to outer magnetic field under the annealing treatment of 150 ${ }^{\circ} \mathrm{C}$, the azimuthal angle $\phi$ between length direction and magnetic field direction refers to domain distribution from $0^{\circ}$ to $360^{\circ}$. Four regions of azimuthal angles of isotropic MS for the samples of annealing temperature of as grown and $150^{\circ} \mathrm{C}$ are $\phi=50^{\circ}-130^{\circ}, \phi=230^{\circ}-310^{\circ}$ and $\phi=-40^{\circ}-40^{\circ}, \phi=140^{\circ}-220^{\circ}$, respectively.

But in case of $100^{\circ} \mathrm{C}$, interlayer coupling field is nearly 0 Oe between $\mathrm{NiFe}(4 \mathrm{~nm})$ which are pinned layer in two free layer with $\mathrm{Cu}$ layer of nonmagnetic that placed in the middle. We can find out that hysteresis property of ultrasoft magnetic as $\mathrm{H}_{\mathrm{c}}$ is less than 1.0 Oe from the minor MR curve as shown in Fig. 6(b). Also, Fig. 6(b) shows that MS determining magnetization properties of highly sensitive element has nearly same value of $1.40 \% / \mathrm{Oe}$. This would be considered to get highly magnetic resistance properties by keeping high MS of isotropy after fine pattern. Also, the angle $\theta$ between a length direction and a magnetic field perpendicular direction at $100{ }^{\circ} \mathrm{C}$ of annealing temperature refers to domain distribution from $0^{\circ}$ to $360^{\circ}$ in Fig. 5. It is one cause that we have lower value of $\mathrm{H}_{\mathrm{c}}$ at $100{ }^{\circ} \mathrm{C}$ than $150{ }^{\circ} \mathrm{C}$. If you change the angle of magnetic field at $45^{\circ}$ domain which consistent magnetic isotropy was represented, we will have isotropic property which can keep consistent MS value regardless of angle of $\phi=-10^{\circ}-100^{\circ}, \phi=170^{\circ}-280^{\circ}$, and $\Delta \phi=110^{\circ}$.

Table 1 shows summary of the magnetoresistance pro- perties and the in-plane isotropic magnetization after three different treatment with post annealing condition for the IrMn based dual-type GMR-SV film. For one dual-type GMR-SV samples so called to the name 130130A, two different post annealing temperatures are $100{ }^{\circ} \mathrm{C}$ and 150 ${ }^{\circ} \mathrm{C}$ during the annealing time of $1 \mathrm{hr}$, and the rotation angles of $90^{\circ}$. The properties for sample $130130 \mathrm{~A}$ of as grown, $100{ }^{\circ} \mathrm{C}$, and $150{ }^{\circ} \mathrm{C}$ are the MR ratio of $6.0 \%-6.5$ $\%$, the MSs of $1.35 \% / \mathrm{Oe}, 1.4 \% / \mathrm{Oe}$, and $1.3 \% / \mathrm{Oe}$, and the $\mathrm{H}_{\mathrm{c}} \mathrm{s}$ of $3.0 \mathrm{Oe}, 1.0 \mathrm{Oe}$, and $3.2 \mathrm{Oe}$, respectively. As other major property, the in-plane isotropic magnetizations show one regional distributions having the azimuthal angles for the MS. The regions of azimuthal angles of isotropic MS for the samples of annealing temperature of $100^{\circ} \mathrm{C}$ are $-10^{\circ}-100^{\circ}$, and $\Delta \phi=110^{\circ}$. On other side, The regions of azimuthal angles of isotropic MS for the samples of annealing temperature of as grown and $150{ }^{\circ} \mathrm{C}$ are $50^{\circ}-130^{\circ},-40^{\circ}-40^{\circ}$, and $\Delta \phi=80^{\circ}$.

Even if magnetic beads which are less than $1 \mu \mathrm{m}$ would be super paramagnetic, we can predict that there would be very tiny magnetic field in random direction. Because it would be very weak magnetic field of isotropy, in this case GMR-SV element which was made after free layer and pinned layer to be crossed would keep above $1.0 \% /$ Oe of limited isotropy MS between $-10^{\circ}$ to $110^{\circ}$ like thin film . It will be considered to enable to measure magnetic beads. Because it is important to control the magnetic property whose particles have in the study of magnetism nanoparticle.

Generally, we can compare the power of magnetic field of magnetic bead measuring susceptibility with magnetic nanoparticle coated with silica and magnetite without coating. The measurement result of susceptibility according to mole density of silica precursor refer that susceptibility are proportional to specific surface area and have an inverse relationship with size. Therefore, we can find out that the value of MS, residual magnetization which are

Table 1. The magnetoresistance properties and the in-plane isotropic magnetization as grown sample and after two different treatment samples with post annealing temperatures of as grown, $100{ }^{\circ} \mathrm{C}$, and $150{ }^{\circ} \mathrm{C}$ for the $\mathrm{IrMn}$ based dual-type GMR-SV thin film by using sample name of 130130A.

\begin{tabular}{cccc}
\hline \hline & Sample name : $130130 \mathrm{~A}$ & & \\
\hline Annealing temperature & As grown & $100^{\circ} \mathrm{C}$ & $150^{\circ} \mathrm{C}$ \\
Rotational angle $(\theta)$ & - & $90^{\circ}$ & $90^{\circ}$ \\
Induced Easy-axis & Width direction & $45^{\circ}$ & Length direction \\
Magnetoresistance ratio (MR) & $6.5 \%$ & $6.0 \%-6.4 \%$ & $6.0 \%-6.3 \%$ \\
Magnetic sensitivity (MS) & $1.3 \% / \mathrm{Oe}$ & $1.4 \% / \mathrm{Oe}$ & $1.3 \% / \mathrm{Oe}$ \\
Coercivity $\left(\mathrm{H}_{\mathrm{c}}\right.$ ) of free FM layer & 3.0 Oe & $1.0 \mathrm{Oe}$ & $3.2 \mathrm{Oe}$ \\
Isotropic MS azimuthal angle $(\phi)$ & $50^{\circ}-130^{\circ}$ & $-10^{\circ}-100^{\circ}$ & $-40^{\circ}-40^{\circ}$ \\
\end{tabular}


distinguishable at $1.3 \% / \mathrm{Oe}-1.5 \% / \mathrm{Oe}$ keep the properties of super paramagnetism with the size of $30 \mathrm{emu} / \mathrm{g}-70$ $\mathrm{emu} / \mathrm{g}$.

Our experimental results show minor MR curves that have AMR property linearly proportional to a fine magnetic field change according to outside, it can also be applied to bio-element easily detected magnetic particle as well as magnetic field sensitivity to be highly improved. In other words, as fine magnetic field to get increased a high MR ratio and MS around 0 Oe where $\mathrm{H}_{\mathrm{c}}$ of free layer from dual type GMR-SV film minimized, it aims to obtain the temperature condition of an optimized annealing treatment. For the main property that GMR-SV element would be used as an extremely fine detection and bio-element of magnetic field, it is considered to have linear sensitivity and high output value.

The uniaxial magnetic anisotropy of ferromagnetic pinned layer and free layer surely cross each other in surface considered to be fine patterning element. MR property should be eliminated the effect of AMR and had a high output value in the effect of isotropic linear MS. To develop all of elements through vacuum annealing treatment, we can get to know it would be needed post annealing treatment process which change easy axis into hard axis at $100{ }^{\circ} \mathrm{C}$ after optical lithography process.

\section{Conclusion}

We implemented post annealing treatment in vacuum chamber to control easy axis in surface of thin film sample which has dual-type GMR-SV fabricated by using $\mathrm{dc}$ magnetron deposition system. The multilayer structure of dual-type GMR-SV is $\mathrm{Ta}(5 \mathrm{~nm}) / \mathrm{NiFe}(8 \mathrm{~nm}) / \mathrm{Cu}(2.3$ $\mathrm{nm}) / \mathrm{NiFe}(4 \mathrm{~nm}) / \mathrm{IrMn}(5 \mathrm{~nm}) / \mathrm{NiFe}(4 \mathrm{~nm}) / \mathrm{Cu}(2.3 \mathrm{~nm}) /$ $\mathrm{NiFe}(8 \mathrm{~nm}) / \mathrm{Ta}(5 \mathrm{~nm})$ which uniaxial anisotropy magnetic field induced during the deposition of film. Inside of vacuum chamber, a dual-type GMR-SV film increased temperature to $150{ }^{\circ} \mathrm{C}$ of post annealing temperature and induced to change the easy axis of magnetization of the ferromagnetic substance in the plan of film. The optimized annealing temperature to induce the magnetization easy axis of a magnetic layer rotation in surface was $100{ }^{\circ} \mathrm{C}$. The MR ratio, coercivity, and MS of MR curve are determined by a magnetic isotropy domain distribution depending on rotating condition of self-domain of magnetic layer.

We measured a MR curve for the dual-type GMR-SV film with a measuring direction variation of the external magnetic field from $\phi=0^{\circ}$ to $\phi=360^{\circ}$ angles. At the annealing temperature of $100{ }^{\circ} \mathrm{C}$, by switching process of magnetization direction in the surface of thin film, we can get a magnetic isotropy property of MR ratio $6.0 \%-6.4$ $\%, \mathrm{H}_{\mathrm{c}}$ of $1.0 \mathrm{Oe}$, MS of around $1.4 \% / \mathrm{Oe}$, in an isotropic magnetization regional distribution of $\phi=-10^{\circ}-100^{\circ}$, and $\Delta \phi=110^{\circ}$. So a highly sensitivity GMR-SV thin film element crossing surface of ferromagnetic pinned layer and free layer would have possibility to be used as a highly sensitive biosensor which can detect micro magnetic bead in random direction.

\section{Acknowledgement}

This work was supported by the National Research Foundation of Korea (NRF) funded by the Korea government (Ministry of Education) with the Grant No. of NRF2016R1D1A1B03936289.

\section{References}

[1] Y. Laosiritaworn and W. Laosiritaworn, J. Magn. 31, 11 (2015).

[2] S. H. Hong, Physics and High Technology 18, 16 (2009).

[3] D. A. Baker, Nature 405, 39 (2000).

[4] S. X. Wang and A. M. Taratorin, Magnetic Information Storage Technology, San Diego, USA. Academic Press (1999).

[5] B. S. Chun and J. S. Jeong, J. Magn. 16, 328 (2011).

[6] P. Robert and C. B. Roger, Bioelectricity - A Quantitative Approach, 3rd ed. Springer, (2007) Chap. 7.

[7] G. Li, S. Sun, R. J. Wilson, R. L. White, N. Pourmand, and S. X. Wang, Sens. Actuators. A 126, 98 (2006).

[8] H. S. Whang, S. J. Yun, J. Moon, and S. B. Choe, J. Magn. 31, 8 (2015).

[9] D. L. Graham, H. A. Feliciano, P. P. Fretias, L. A. Clarke, and M. D. Amaral, Sens. Actuators: B 107, 936 (2005).

[10] B. M. de Boer, J. A. H. M. Kahlman, T. P. G. H. Jansen, H. Duric, and J. Veen, Biosens. Bioelectron. 22, 2366 (2006).

[11] W. H. Lee, D. G. Hwang, and S. S. Lee, J. Magn. 14, 18 (2009).

[12] P. Khajidmaa, K. J. Park, and S. S. Lee, J. Korean Magn. Soc. 23, 98 (2013).

[13] P. Khajidmaa and S. S. Lee, J. Korean Magn. Soc. 23, 193 (2013).

[14] J. V. Kim and R. L. Stamps, Phys. Rev. B 71, 094405 (2005).

[15] M. Fecioru-Morariu, Exchange bias in metallic ferro-/ antiferromagnetic bilayers. Effects of structure, dilution, anisotropy and temperature, Ph.D. thesis: Institute of Physics of RTWH Aachen. (2008). 